\title{
Anomalous reflection of visible light by all-dielectric gradient metasurfaces
}

\author{
Nikolaos L. Tsitsas ${ }^{1, *}$ and Constantinos A. Valagiannopoulos ${ }^{2}$ \\ ${ }^{1}$ Department of Informatics, Aristotle University of Thessaloniki, Thessaloniki GR-54124, Greece \\ ${ }^{2}$ Department of Physics, School of Science and Technology, Nazarbayev University, 53 Qabanbay Batyr Ave., Astana KZ-010000, Kazakhstan \\ ${ }^{*}$ Corresponding author: ntsitsas@csd.auth.gr
}

Received 31 January 2017; accepted 12 February 2017; posted 16 February 2017 (Doc. ID 285933); published 15 March 2017

\begin{abstract}
Plane wave scattering by a planar metasurface composed of two periodically alternating rectangular dielectric rods is considered. A rigorous integral equation methodology is employed for the analysis and the accurate determination of the reflected and transmitted fields. Systematic optimizations with respect to the configuration's parameters are performed, which reveal that it is possible to obtain significantly enhanced anomalous reflection (with simultaneously suppressed ordinary reflection predicted by Snell's law) with power varying from $92 \%$ to almost $100 \%$ of the input one, depending on the color of the incident light. It is shown that these reflection properties are supported by metasurfaces easily realizable with specific low-loss dielectric materials. In this way, several all-dielectric optimal designs are reported that can be used in numerous applications demanding anomalous reflection in the visible range. () 2017 Optical Society of America
\end{abstract}

OCIS codes: (050.0050) Diffraction and gratings; (160.3918) Metamaterials; (240.3695) Linear and nonlinear light scattering from surfaces.

\section{INTRODUCTION}

Gradient metasurfaces have remarkable properties with respect to manipulating electromagnetic waves including controlling the propagation direction of an incident wave over subwavelength distances, modifying the optical wavefront, bending and focusing light, generating anomalous reflection and refraction phenomena as well as desired distributions of electromagnetic waves [1-10]. Metamaterial gradient index diffraction gratings, composed of metallic and dielectric parts, are utilized in gradient index optics applications [11], in multiband electromagnetic absorbers [12], and in the improvement of near-field optical enhancement [13]. Recently, all-dielectric gradient metamaterials have received particular attention and have been shown to assist significantly in subdiffraction confinement and guiding of light without metals [14], in achieving very high transmission efficiency $[15,16]$, in electromagnetic mode conversion [17], and in controllable coloring [18]. Such artificial structures are realized by using low-loss dielectric materials in building alternating blocks of transparent and high-refractiveindex media.

Among all the aforementioned interesting effects recorded with use of gradient metasurfaces, special attention will be paid to anomalous reflection. Such terminology is used to describe unusual and remarkable steering of the incident illumination toward directions not predicted by Snell's law. By properly designing the texture of the metasurface, one can achieve reflection of the incident electromagnetic field into free space in counterintuitive ways being very useful in numerous applications. In particular, anomalous reflection is employed in fabrication of ultraefficient antireflection coatings [19], while the construction of polarization and three-dimensional beam splitters becomes easier by obtaining controllable out-of-plane refraction [20]. The significance of anomalous reflection is not confined only in components operating at the visible or the infrared part of the frequency spectrum; it has been utilized for microwave surface plasmon coupling [21] or even for acoustic wavefront engineering [22]. Putting it to a more general basis, anomalous reflection is a fundamental mechanism that decisively assists in tuning [18], directing [1], and steering [23] the electromagnetic waves. Therefore, it is worthy of further examination and study.

In this work, we investigate plane wave scattering by a twodimensional, planar structure containing periodically alternating rectangular rods of two different dielectrics. A rigorous and highly accurate entire-domain integral equation methodology is employed for the analysis of the associated scattering and diffraction phenomena by such a metasurface. The methodology was initially introduced and developed in [24] for the analysis of dielectric grating structures and was subsequently extended to the scattering analysis of metasurfaces in [25]. We focus on the determination of suitable geometrical and electromagnetic parameters of the metasurface in order for it to exhibit 
significant anomalous reflection. In particular, it is possible, due to the inhomogeneous nature of the structure (gradient index), to guide the major part of the diffracted power toward the same region of the plane that the metasurface is excited (directions of the -1 order in reflection and transmission), unlike what is happening is the case of a homogeneous slab (propagation of only the zero order dictated by Snell's law). Our primary aim is to stimulate further experimental as well as theoretical works by reporting various simple and realistic grating configurations comprised of ordinary dielectric media, which at specific visible frequency bands give substantial anomalous reflection with simultaneously suppressed and annihilated zero-order responses.

Following a certain systematic optimization scheme, we show that metasurfaces composed of conventional low-loss dielectric materials with specifically designed geometrical characteristics can reflect for all the examined frequencies in the visible range more than $92 \%$ of the incident field's power back to the direction where the incident field propagates. It is worth noting that for certain colors, the reflected powers of the -1 order reach almost $100 \%$. For each optimized configuration, we also examine in detail the variations of the reflected and transmitted powers of the -1 order with respect to the angle of incidence as well as the operating wavelength.

In the subsequent analysis, an $\exp (i \omega t)$ time dependence of the field quantities is assumed and suppressed, with $\omega=2 \pi /\left(\lambda_{0} \sqrt{\varepsilon_{0} \mu_{0}}\right)$ as the angular frequency, $t$ as time, and $i=\sqrt{-1}$. The permittivity, permeability, and wavelength of free space are denoted by $\varepsilon_{0}, \mu_{0}$, and $\lambda_{0}$, respectively.

\section{MATHEMATICAL ANALYSIS OF THE SCATTERING PROBLEM}

Consider the gradient dielectric metasurface, depicted in Fig. 1, which is composed of a dielectric slab with refractive index $n_{1}$ and thickness $w$ containing $\Lambda$-periodic rectangular inclusions of refractive index $n_{2}$, width $s \Lambda$, and same thickness $w$. Thus, the metasurface under investigation is a $\Lambda$-periodic structure with two dielectric materials alternating inside each unit cell. The semi-infinite cover and substrate plane regions, above and below the slab, are characterized by refractive index $n_{0}$ (for vacuum, we have $n_{0}=1$ ). The entire structure has permeability $\mu_{0}$ and is assumed uniform along the direction $\hat{\mathbf{y}}$.

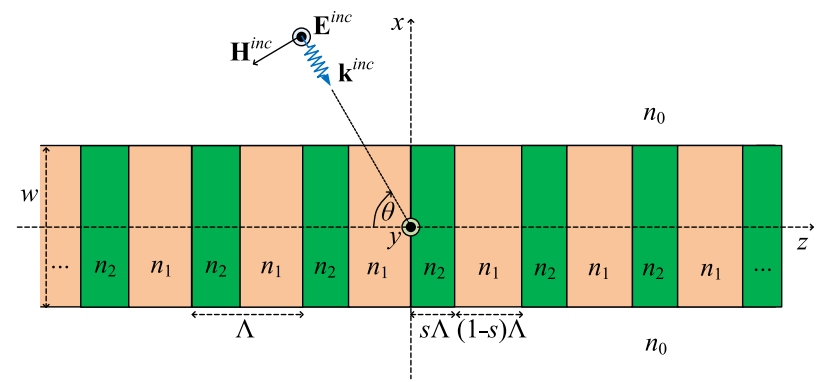

Fig. 1. The considered all-dielectric $\Lambda$-periodic metasurface composed of two dielectric materials with refractive indices $n_{2}$ and $n_{1}$, widths $s \Lambda$ and $(1-s) \Lambda$, and common thickness $w$. The metasurface is excited by a unit amplitude TE-polarized plane wave with angle of incidence $\theta$.
The metasurface is excited by a transverse-electric- (TE) polarized plane wave with angle of incidence $\theta$ (see Fig. 1) and electric field given by

$$
\mathbf{E}^{i}(x, z)=\Psi^{i}(x, z) \hat{\mathbf{y}}=\exp \left[i k_{0} n_{0}(\sin \theta x-\cos \theta z)\right] \hat{\mathbf{y}},
$$

where $k_{0}=\omega \sqrt{\varepsilon_{0} \mu_{0}}$ denotes the free-space wavenumber.

The total electric field induced in every region of the scattering problem is of the form $\mathbf{E}(x, z)=\Psi(x, z) \hat{\mathbf{y}}$, and the unknown scalar electric field factor $\Psi$ admits the integral representation [24-27]

$$
\begin{aligned}
\Psi(x, z)= & \Psi_{0}(x, z)+k_{0}^{2}\left(n_{2}^{2}-n_{1}^{2}\right) \iint_{S} G\left(x, z ; x^{\prime}, z^{\prime}\right) \Psi\left(x^{\prime}, z^{\prime}\right) \\
& \times \mathrm{d} x^{\prime} \mathrm{d} z^{\prime},(x, z) \in \mathbb{R}^{2},
\end{aligned}
$$

where $\Psi_{0}$ and $G$ are the electric field factor and the Green's function both induced on the respective structure in the absence of the inclusions due to the plane incident wave of Eq. (1) and a line-source excitation inside the dielectric slab, respectively. Furthermore, $S$ denotes the total transverse cross section of the rectangular inclusions. Functions $\Psi_{0}$ and $G$ have been analytically determined (for more complicated geometries) in [24,25].

According to the Floquet-Bloch theorem [28], the electric field factor is expressed as

$$
\Psi(x, z)=\exp \left(-i k_{0} n_{0} \cos \theta z\right) u(x, z),
$$

where $u(x, z)$ is a $\Lambda$-periodic function of $z$. By reducing the double integrals of Eq. (2) to integrals on the inclusion's cross section on the basic unit cell $[0, s \Lambda] \times\left[-\frac{w}{2}, \frac{w}{2}\right]$ and by using the Poisson's summation formula for the Dirac function [29], we reformulate the integral representation in Eq. (2) with respect to function $u$ as

$$
\begin{aligned}
u(x, z)= & \Psi_{0}(x, z) \exp \left(i k_{0} n_{0} \cos \theta z\right) \\
& +\frac{k_{0}^{2}\left(n_{2}^{2}-n_{1}^{2}\right)}{2 \Lambda} \sum_{p=-\infty}^{+\infty}\left[\exp \left(-i \frac{2 \pi p}{\Lambda} z\right)\right. \\
& \left.\times \int_{0}^{s \Lambda} \int_{-\frac{w}{2}}^{\frac{w}{2}} u\left(x^{\prime}, \zeta^{\prime}\right) \exp \left(i \frac{2 \pi p}{\Lambda} \zeta^{\prime}\right) \gamma_{p}\left(x, x^{\prime}\right) \mathrm{d} x^{\prime} \mathrm{d} \zeta^{\prime}\right],
\end{aligned}
$$

where function $\gamma_{p}$ denotes the kernel of the determined Fourier integral expression of the Green's function $G$ (see Appendix A of [25]).

Next, we apply to Eq. (4) a highly efficient entire-domain Galerkin technique. Function $u(x, z)$ is expanded on the cross section of the inclusion inside the basic unit cell in the following Fourier series with respect to $z$ :

$$
\begin{aligned}
u(x, z)= & \sum_{n=-\infty}^{+\infty}\left[c_{n}^{+} \exp \left(g_{n} x\right)+c_{n}^{-} \exp \left(-g_{n} x\right)\right] \\
& \times \exp \left(-i \frac{2 \pi n}{\Lambda} z\right),
\end{aligned}
$$

where $g_{n}=\sqrt{\left(k_{0} n_{0} \cos \theta+\frac{2 \pi n}{\Lambda}\right)^{2}-k_{0}^{2} n_{2}^{2}}$, while $c_{n}^{ \pm}$are determinable coefficients. The $x$-dependent Fourier coefficients in Eq. (5) are referred to, in the terminology of the method of moments, as the electric field entire-domain expansion (basis) 
functions. Then, we restrict the observation vector $(x, z)$ in Eq. (4) on the domain of the inclusion, and hence function $u$ in Eq. (4) is expanded in the Fourier series of Eq. (5). Furthermore, by considering the inner products of both sides of the resulting equation with the test functions [which are the conjugates of the expansion functions in Eq. (5)] and carrying out the integrations analytically, we formulate an algebraic infinite nonhomogeneous square linear system of equations with respect to the unknown coefficients $c_{n}^{ \pm}, n \in \mathbb{Z}$. This infinite system is solved numerically by truncation as follows: we take into account the entire-domain expansion as well as the test functions with maximum absolute order $N_{t}$ and reduce the infinite system to a truncated square linear system of order $4 N_{t}+2$ of the form

$$
\left[\begin{array}{cc}
\mathbf{A}^{++} & \mathbf{A}^{+-} \\
\mathbf{A}^{-+} & \mathbf{A}^{--}
\end{array}\right]\left[\begin{array}{l}
\mathbf{c}^{+} \\
\mathbf{c}^{-}
\end{array}\right]=\left[\begin{array}{l}
\mathbf{b}^{+} \\
\mathbf{b}^{-}
\end{array}\right]
$$

where $\mathbf{c}^{ \pm}$are the $\left(2 N_{t}+1\right)$ column vectors containing the unknown coefficients $c_{n}^{ \pm}$, while $\mathbf{A}^{ \pm \pm}$and $\mathbf{b}^{ \pm}$are square matrices of order $\left(2 N_{t}+1\right)$ and $\left(2 N_{t}+1\right) \times 1$ column vectors, respectively; their elements are given in [24] and [25].

After having determined the coefficients $c_{n}^{ \pm}$by solving the system in Eq. (6), the reflected and transmitted electric field factors are, respectively, expressed by

$$
\begin{array}{cc}
\Psi^{r}(x, z)=\sum_{p=-\infty}^{+\infty} r_{p} \exp \left[i\left(k_{x, p} x-k_{z, p} z\right)\right], & x>\frac{w}{2}, z \in \mathbb{R}, \\
\Psi^{t}(x, z)=\sum_{p=-\infty}^{+\infty} t_{p} \exp \left[-i\left(k_{x, p} x+k_{z, p} z\right)\right], & x<-\frac{w}{2}, z \in \mathbb{R},
\end{array}
$$

with $r_{p}$ and $t_{p}$ denoting, respectively, the complex reflection and transmission coefficients, which are explicitly determined by means of $c_{n}^{ \pm}$. Moreover, $k_{x, p}$ and $k_{z, p}$ denote the components of the reflected and transmitted wave vectors and are given by

$$
\begin{aligned}
& k_{x, p}=i \sqrt{\left(k_{0} n_{0} \cos \theta+\frac{2 \pi p}{\Lambda}\right)^{2}-k_{0}^{2} n_{0}^{2}}, \\
& k_{z, p}=k_{0} n_{0} \cos \theta+\frac{2 \pi p}{\Lambda} .
\end{aligned}
$$

Each term in the field series in Eqs. (7) and (8), indexed by $p \in \mathbb{Z}$, represents a specific field component and will be referred to as the $p$-reflected or $p$-transmitted diffracted order. According to Eq. (9), the diffracted orders, which propagate along the $x$ axis, depend on the period $\Lambda$, the free-space wavelength $\lambda_{0}$, the refractive index $n_{0}$, and the angle of incidence $\theta$. It is important to analyze the conditions for which a diffracted order $p$ is propagating or becomes evanescent. The zero-order reflected and transmitted fields are always propagating (Snell's law). For orders $p \neq 0$, we define the thresholds

$$
p^{ \pm}= \pm \frac{\Lambda}{\lambda_{0}} n_{0}(1 \mp \cos \theta)
$$

for which the diffracted fields switch from propagation to cutoff. More precisely, when $p^{-}<p<p^{+}$, the wave vector component $k_{x, p}$ is real, and thus the $p$-order reflected and transmitted fields are propagating along the $x$ axis. On the other hand, when $p>p^{+}$or $p<p^{-}$, the component $k_{x, p}$ becomes purely imaginary and the $p$-order fields are evanescent.

The presented entire-domain integral equation methodology is rigorous and provides semi-analytical solutions with high numerical stability, controllable accuracy, and high efficiency. The Green's function is analytically expressed as a Fourier integral and all the involved integrations are analytically carried out. Therefore, the method is very efficient in terms of CPU time and computer memory and the sole approximation in the solution is the final truncation of the expansion functions sets. All these advantages render the proposed technique very suitable for optimization of multiparametric structures requiring detailed scanning of the parameter space, like the one analyzed below.

The required truncation order $N_{t}$ is determined by applying a convergence control to the solutions for increasing $N_{t}$ according to the following two criteria, in consistency with the literature [30,31]: (i) an energy conservation condition (reflected and transmitted fields must conserve power within 1 part in $10^{8}$ ) and (ii) convergence to the solution with increasing $N_{t}$ for all the inclusion's and the incident wave's parameters. Representative convergence patterns of the zero-order reflection coefficient, depicted in [24] and [25], show that small values of $N_{t}$ provide sufficient convergence, a fact which constitutes a basic advantage of the method. For all the results presented hereafter, it has been checked that the choice $N_{t}=5$ is sufficient so that both criteria (i) and (ii) are satisfied. This superior numerical efficiency of the present method is mainly justified due to that the unknown electric field and the entire-domain expansion functions satisfy the same physical law, i.e., the Helmholtz equation, and that the utilized background's Green's function satisfies inherently the boundary conditions in the inclusion-free structure.

A detailed study concerning convergence and numerical efficiency aspects of the integral equation methodology is included in [24], where the method is also validated by obtaining coincident results with different methodologies which however require a significantly larger number of basis functions.

\section{PARAMETER SELECTION AND OPTIMIZATION APPROACH}

Our major purpose in this work (as already mentioned in Section 1 above) is to investigate suitable selections of parameters of the metasurface in order for it to generate the so-called anomalous reflection and transmission phenomena in which cases the main contribution in the reflected and transmitted fields comes from waves traveling along directions not predicted by Snell's law. In such cases, most of the electromagnetic response of the structure is confined to directions lying in the same region of space with the incoming field, thereby forcing light to propagate toward the side of incidence.

The metasurface in Fig. 1 is considered as free-standing. Therefore, the refractive index of the background medium (vacuum) is taken as equal to unity: $n_{0}=1$.

\section{A. Parameter Selection}

We require that the examined metasurfaces admit only the zeroand the -1 -reflection and transmission orders to propagate. 
This implies that $p^{+}<1$ and $-2<p^{-}<-1$, which lead by Eq. (11) to the following conditions:

$$
\max \left\{1-\cos \theta, \frac{1+\cos \theta}{2}\right\}<\frac{\lambda_{0}}{\Lambda}<1+\cos \theta .
$$

Moreover, in order for the -1 order to be reflected back in the same (second) quadrant of the plane with that where the incident field propagates and impinges on the metasurface, as well as to be transmitted back in the corresponding (third) quadrant, it must hold that $k_{z,-1}<0$, which by Eq. (10) implies

$$
\cos \theta<\frac{\lambda_{0}}{\Lambda} .
$$

The latter is satisfied for all $\theta$ when $\lambda_{0}>\Lambda$ and for essentially all $\theta$ when $\lambda_{0} \lesssim \Lambda$. Since $\frac{1+\cos \theta}{2}>\cos \theta$, the condition in Eq. (12) implies the condition in Eq. (13) and accordingly we proceed only with Eq. (12).

The angle between the negative $z$ axis and the wave vector of the -1 -reflected order (measured clockwise as the angle $\theta$ in Fig. 1) is given by

$$
\theta_{-1}^{r}=\arctan \left(\frac{\sqrt{1-\left(\cos \theta-\frac{\lambda_{0}}{\Lambda}\right)^{2}}}{\cos \theta-\frac{\lambda_{0}}{\Lambda}}\right) .
$$

The respective angle corresponding to the -1 -transmitted order has the same value with $\theta_{-1}^{r}$ and is measured counterclockwise from the negative $z$ axis. Figure 2 depicts the contour plot of the absolute value of the difference between the angle $\theta_{-1}^{r}$ of the -1 -reflected order and the angle of incidence $\theta$ as function of $\theta$ and the grating's electrical period $\Lambda / \lambda_{0}$. The respective information for the -1-transmitted order is also depicted in Fig. 2 since, as mentioned above, the propagation direction of the -1-transmission order is symmetric with respect to the $z$ axis to that of the -1 -reflected order. Blank (white) regions in Fig. 2 correspond to pairs of $\theta$ and $\Lambda / \lambda_{0}$ that do not fulfill any of the conditions in Eqs. (12) and (13) (namely, propagation of only two diffracted orders and reflection of the -1 order to the same quadrant where the incident field propagates). From Fig. 2, we can conclude the

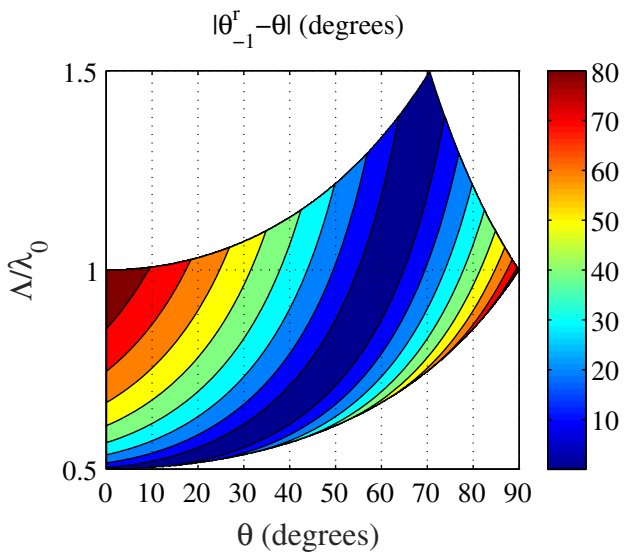

Fig. 2. Contour plot of $\left|\theta_{-1}^{r}-\theta\right|$ as a function of $\theta$ and $\Lambda / \lambda_{0}$. range of values of the incidence angle $\theta$, which is meaningful and useful to select in order to properly investigate anomalous reflection and transmission effects. This range corresponds to the dark blue region of Fig. 2, where the difference between $\theta_{-1}^{r}$ and $\theta$ is relatively small (precisely does not exceed $8^{\circ}$ ). Moreover, in the vicinity of the angle $\theta$ that we will select, we need to have a sufficient range of values of $\Lambda / \lambda_{0}$, satisfying Eq. (12), in order to be able to have a larger parametric space for the subsequent optimizations. It is thus meaningful to make an indicative selection of $\theta=60^{\circ}$; similar investigations and optimizations with those reported below can be performed for alternative choices of incidence angles $\theta$.

Up to this point, we have considered a metasurface admitting only the zero- and the -1 -diffracted orders, explained the range of $\lambda_{0} / \Lambda$ that the latter consideration implies, and have justified the choice of the angle of incidence. As the next step, we elaborate on proper and relevant selections for the remaining parameters of the problem, namely, the operating wavelength $\lambda_{0}$, the refractive indices $n_{1}$ and $n_{2}$, the thickness $w$ of the dielectric slab, and the duty cycle $s$ of the inclusions. We consider five different operating wavelengths $\lambda_{0}$, each one corresponding to a different color of the visible spectrum; more precisely, we take the value of $\lambda_{0}$ at the central wavelength in the regime of each color (as defined by [32]). Other values of $\lambda_{0}$ might also be considered. For each $\lambda_{0}$ and for constant $\theta=60^{\circ}$, the range of the period $\Lambda$ of the metasurface is that dictated by Eq. (12). For the refractive index of the dielectric slab, we consider that $n_{1} \cong 1.35$. Representative materials with such refractive index values in almost the entire visible range are Teflon AF fluoropolymers [33]. For the inclusion, we assume that it is occupied by lossless silicon and set the values of the inclusion's refractive index $n_{2}$ for each color in the visible range according to the classical frequency-varying models of $[34,35]$. Concerning the thickness $w$ of the dielectric slab, the general requirement is to be suitably small, permitting the description as a metasurface. In the subsequent results, the optimal values of $w$ will actually turn out to be of the order of a few hundred nanometers, which is much smaller than a single effective wavelength into the employed media. The duty cycle $s$ of the inclusions is kept constant, hereafter, at $s=1 / 2$. Thus, the metasurface has the form of a classical binary grating [36]; fabricating a binary grating is easier than other types of gratings like multilevel or graded-index ones [37]. Changing the duty cycle, the number of inclusions per unit cell or even their shape might also be worthy of further investigation; the latter characteristics have been shown to play significant roles in the scattering behaviors of such structures (see, e.g., [24] and [38] in the context of sharp resonances exploited in frequency selective filters).

\section{B. Optimization Approach}

The basic optimization scheme and the metrics utilized are described in this subsection. The main aim is to select suitable metasurface parameters such that the powers carried from the -1 order are significantly enhanced while simultaneously the powers carried by the zero order are significantly suppressed. The electromagnetic fields' powers corresponding to the different reflected and transmitted orders are defined in 
the standard way, following [39] (see also the discussions in [40]), as

$$
\begin{aligned}
& P_{p}^{r}=\frac{\left|r_{p}\right|^{2}}{\sin \theta} \sqrt{1-\left(\cos \theta+p \frac{\lambda_{0}}{\Lambda}\right)^{2}}, \\
& P_{p}^{t}=\frac{\left|t_{p}\right|^{2}}{\sin \theta} \sqrt{1-\left(\cos \theta+p \frac{\lambda_{0}}{\Lambda}\right)^{2}} .
\end{aligned}
$$

The latter quantities are usually called the efficiencies of the diffracted orders, which are defined as the ratios of the flux of the Poynting vector associated with the $p$-diffracted order over the corresponding flux of the incident field [39]. It is also evident that $P_{0}^{r}=\left|r_{0}\right|^{2}$ and $P_{0}^{t}=\left|t_{0}\right|^{2}$. Hence, the energy balance criterion takes the form

$$
\sum_{p \in \mathcal{P}}\left(P_{p}^{r}+P_{p}^{t}\right)=1
$$

where $\mathcal{P}$ is the subset of $\mathbb{Z}$ including only the diffracted orders corresponding to propagating waves; in our case $\mathcal{P}=\{-1,0\}$. Equation (17) states that the sum of all efficiencies is equal to unity and has the physical interpretation that the incident energy is equal to the diffracted energy (for lossless media as the ones considered in the presented examples). Note that the evanescent waves are not taken into account since they do not carry energy.

In all the numerical results presented hereinafter, and as explained in Section 2 above, the energy balance criterion in Eq. (17) was tested to be fulfilled up to the order of $10^{-8}$.

As a metric of the degree of anomalous reflection and transmission, we devise the quantity

$$
m=\frac{P_{-1}^{r}+P_{-1}^{t}}{P_{0}^{r}+P_{0}^{t}}
$$

Large values of $m$ indicate an enhanced ability by the considered structure to steer the reflected/transmitted waves in an unusual direction, totally different from that dictated by Snell's law. In other words, $m$ denotes how significant are the powers carried by the waves of the -1-diffracted order as compared to the ones of zero-diffracted order.

According to the discussions of Section 3.A, we fix the parameters as follows: $\lambda_{0}$ to the central wavelengths of the five colors in the visible range, $\theta=60^{\circ}, n_{0}=1, n_{1}=1.35, n_{2}$ to the refractive index of lossless silicon at the examined wavelengths, and $s=1 / 2$. The remaining parameters are the thickness $w$ of the slab and the period $\Lambda$ of the inclusions. The two latter parameters $w$ and $\Lambda$ are the ones with respect to which we perform the optimization procedure in order to maximize the metric $m$. The optimization is greedy and is based on successive computations of the reflection and transmission coefficients by means of the above-described semi-analytical integral equation methodology. The beneficial characteristics of the methodology, described in Section 2, namely, its superior numerical stability, controllable accuracy, and rapid convergence aid substantially the fast and efficient implementation of the optimizations required in order to determine the desired values of $w$ and $\Lambda$ yielding enhanced anomalous reflection and transmission. A representative contour plot of the metric $m$ in the plane of $w$ and $\Lambda$ for the green color, i.e., $\lambda_{0}=532 \mathrm{~nm}$, is depicted

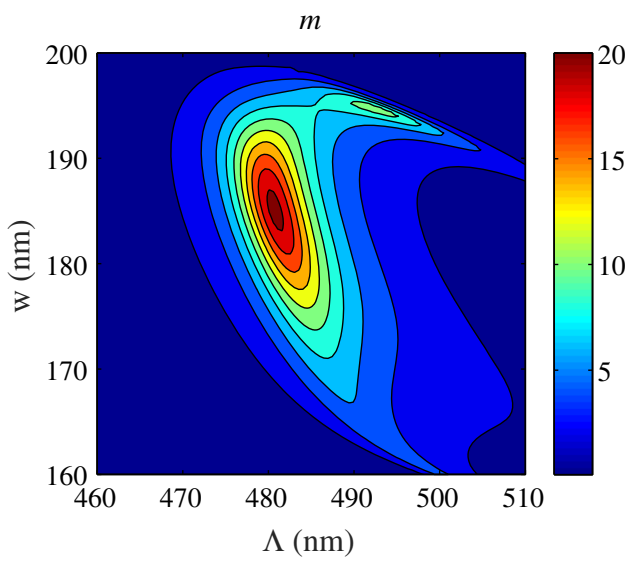

Fig. 3. Contour plot of metric $m$ as function of $w$ and $\Lambda$ for $\lambda_{0}=532 \mathrm{~nm}$ (green).

in Fig. 3. After generating for each color the respective contour plot, like the indicative one in Fig. 3, we proceed to a secondary optimization where we select a narrow interval of $\Lambda$ and then search for the specific value of $w$ in the region of large $m$ (as predicted, e.g., by Fig. 3) so that the values of $P_{-1}^{r}$ and $P_{-1}^{t}$ are significantly larger than those of $P_{0}^{r}$ and $P_{0}^{t}$. The results of this procedure are presented systematically in the next section.

\section{NUMERICAL RESULTS AND DISCUSSION}

Figure 4 depicts the variations of the powers $P_{-1}^{r}, P_{-1}^{t}, P_{0}^{r}$, and $P_{0}^{t}$ versus the inclusion's period $\Lambda$ for the five examined colors in the visible range. For each color, the optimal value of the slab's thickness $w$ was determined according to the secondary optimization scheme described above. The optimal pairs $w$ and $\Lambda$ yielding the maximum reflected power in the -1 order as well as the respective angles $\theta_{-1}^{r}$ are reported for each wavelength in Table 1 . Significantly large values of $P_{-1}^{r}$ are observed for every color, a feature which makes the considered designs very interesting in terms of anomalous reflection. These values start from 0.92 for blue and green light and become almost 1 for yellow, orange, and red light. Moreover, from Table 1 it is noticed that the values of the angle $\theta_{-1}^{r}$ are close to that of the angle of incidence $\theta=60^{\circ}$; they have a $10^{\circ}$ and $7^{\circ}$ deviation for blue and green, respectively, and they are very close to $60^{\circ}$ for yellow, orange, and red. The latter two facts mean that for the selected parameters values, the metasurface exhibits strongly anomalous steering of the incident beam, namely, it reflects back very close to the direction of illumination nearly the entire amount of incident power. The interval of large values of $P_{-1}^{r}$ is wider for blue and green and narrower for yellow, orange, and red, which makes our design immune to fabrication imperfections of the period $\Lambda$. However, as mentioned above, in the cases of the latter three colors, nearly $100 \%$ of the incident field is reflected in the -1 order.

A schematic of the energy balance criterion in Eq. (17) on the map of $P_{0}^{r}+P_{0}^{t}$ and $P_{-1}^{r}+P_{-1}^{t}$ is depicted in Fig. 5(a). For a metasurface composed of lossless materials, feasible values of the obtained powers lie on the line segment joining the points $(0,1)$ and $(1,0)$ on this map. For significant anomalous diffraction, we must obtain a value close to the point 
(a)

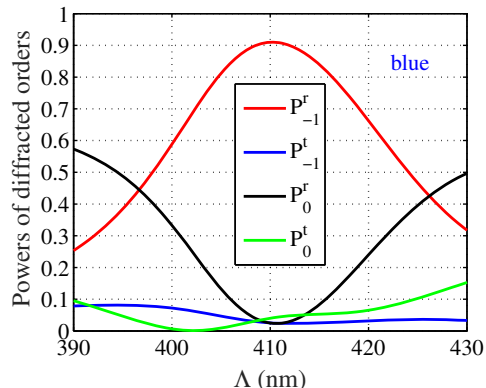

(b)

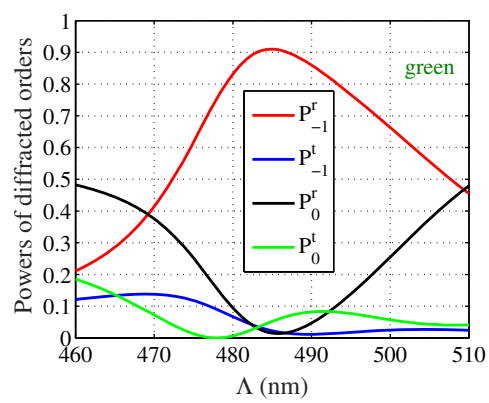

(c)

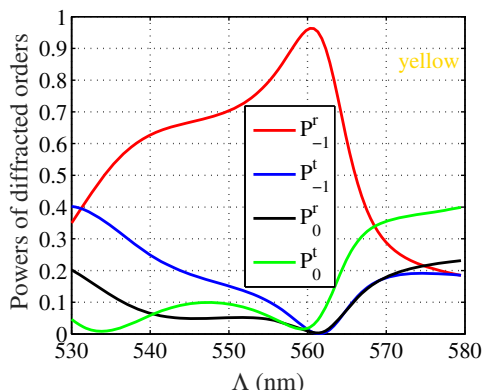

(d)

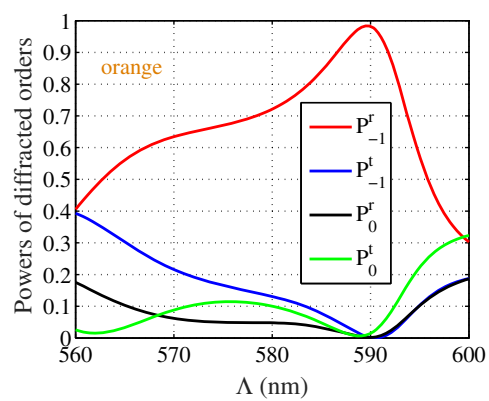

(e)

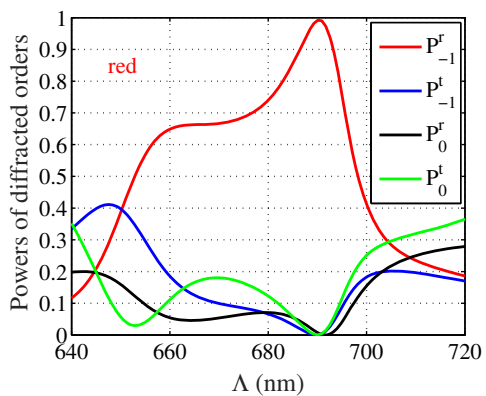

Fig. 4. Powers of -1 - and zero-diffracted orders versus the inclusion's period $\Lambda$ for (a) $\lambda_{0}=470 \mathrm{~nm}$ (blue), (b) $\lambda_{0}=532 \mathrm{~nm}$ (green), (c) $\lambda_{0}=580 \mathrm{~nm}$ (yellow), (d) $\lambda_{0}=605 \mathrm{~nm}$ (orange), and (e) $\lambda_{0}=685 \mathrm{~nm}$ (red)
Table 1. Optimal Pairs of $w$ and $\Lambda$ Yielding Maximum $P_{-1}^{r}$ and Angles $\theta_{-1}^{r}$ for Each Considered Wavelength

\begin{tabular}{lccccc}
\hline Color & $\boldsymbol{\lambda}_{\mathbf{0}}(\mathbf{n m})$ & $\boldsymbol{w}(\mathbf{n m})$ & $\boldsymbol{\Lambda}(\mathbf{n m})$ & $\boldsymbol{P}_{-\mathbf{1}}^{\boldsymbol{r}}$ & $\left.\boldsymbol{\theta}_{-\mathbf{1}}^{\boldsymbol{r}} \mathbf{(}^{\circ}\right)$ \\
\hline Blue & 470 & 139 & 410 & 0.92 & 50 \\
Green & 532 & 178 & 485 & 0.92 & 53 \\
Yellow & 580 & 221 & 561 & 0.97 & 58 \\
Orange & 605 & 234 & 589 & 0.99 & 58 \\
Red & 685 & 274 & 690 & 1 & 60 \\
\hline
\end{tabular}

$\left(P_{0}^{r}+P_{0}^{t}, P_{-1}^{r}+P_{-1}^{t}\right)=(0,1)$. The latter requirement is achieved for all examined colors, as shown in Fig. 5(b), which depicts the attained values of the diffracted orders powers for the metasurface under consideration after implementing the optimization procedure analyzed in Section 3 above. A visual

(a)

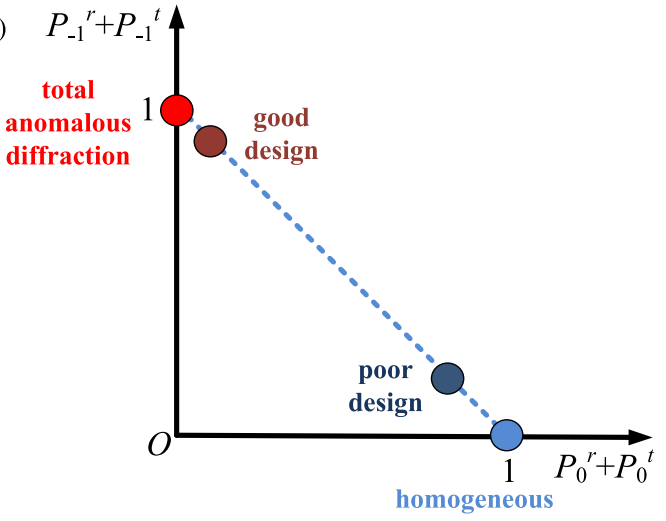

slab

(b)

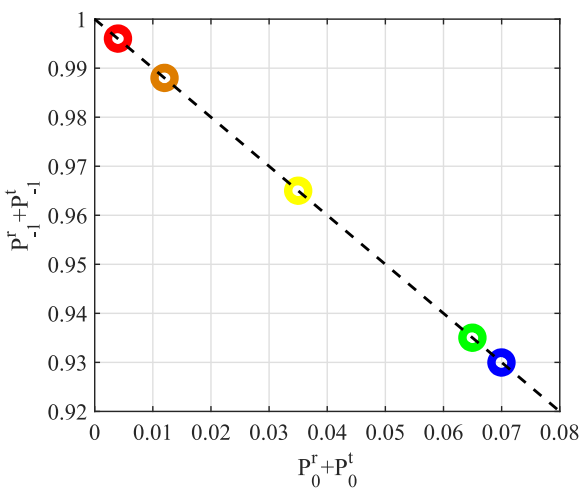

(c)

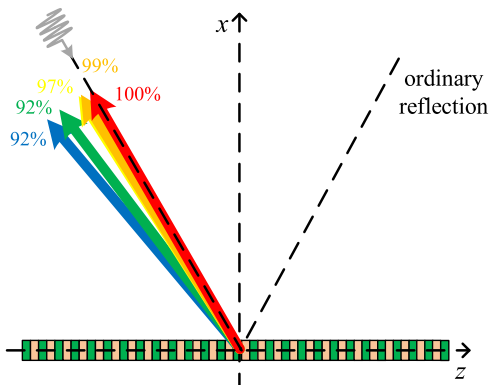

Fig. 5. (a) Schematic of the energy balance criterion for a lossless metasurface supporting two propagating diffracted orders. (b) Optimized values of the diffracted order powers from the metasurface under consideration for each color of visible light. (c) Visual representation of the diffraction performance for the different colors, according to the data of Table 1 . 
representation of the data contained in Table 1, exhibiting the obtained optimized diffraction performance for the different colors, is depicted in Fig. 5(c). In the same figure we show the

(a)

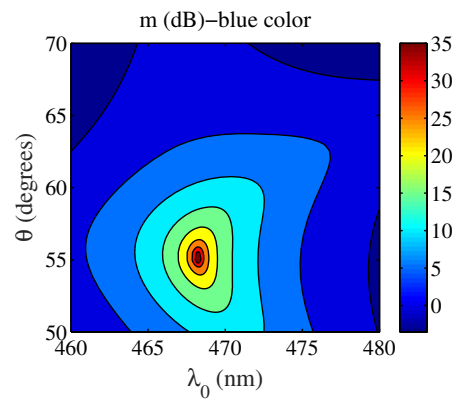

(b)

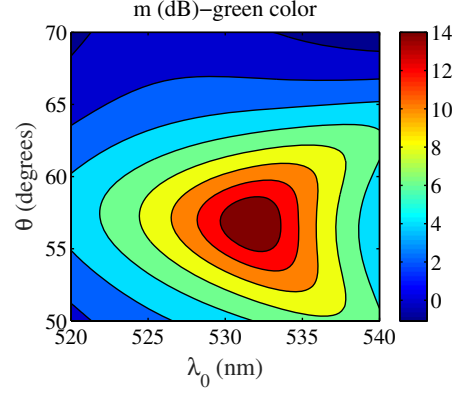

(c)

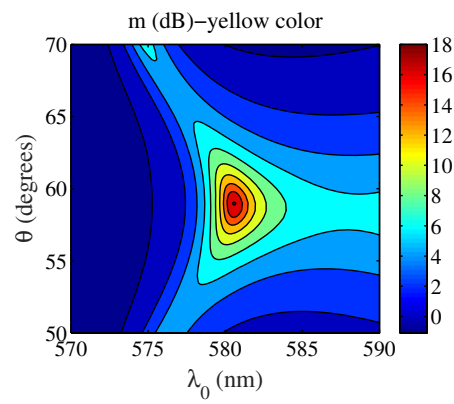

(d)

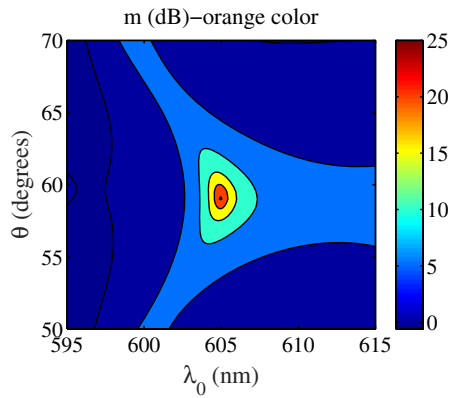

(e)

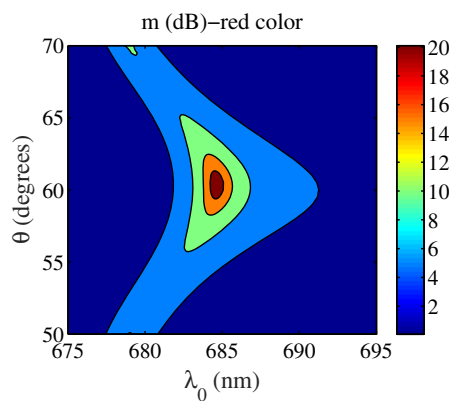

Fig. 6. Contour plots of metric $m$ in $\mathrm{dB}$ as a function of the wavelength $\lambda_{0}$ and the angle of incidence $\theta$ for the optimal values of $w$ and $\Lambda$ of Table 1 . different angles of propagation of the diffracted orders as well as that of ordinary reflection, which is dictated by Snell's law.

Furthermore, for each of the five optimal designs presented above, we represent in Fig. 6 on the $\left(\lambda_{0}, \theta\right)$ map the variations of the metric $m$ in the vicinity of the central operating wavelength (20 nm range) and the angle of incidence, which is kept fixed at $60^{\circ}\left(20^{\circ}\right.$ range). The values of $w$ and $\Lambda$ were fixed to those of Table 1 , corresponding to the maximization of $P_{r}^{-1}$. Extremely large values of $m$ are highly concentrated for almost all examined cases in relatively narrow regions in the plane of $\lambda_{0}$ and $\theta$. The decibel $(\mathrm{dB})$ scale utilized in Fig. 6 emphasizes the large magnitudes of these values; notice that the representation of $m$ in Fig. 6 is scaled differently from the indicative plot of optimization of $m$ in Fig. 3. Therefore, most proposed devices give highly selective anomalous reflection both with respect to the frequency and the direction of excitation, a property very useful in switching applications. On the contrary, our optimal configuration for green light (which exhibits the poorer performance from all proposed designs) retains a substantial $m$ for a wide region on $\left(\lambda_{0}, \theta\right)$ plane, which means that it can reflect in an anomalous way wideband and spatially modulated signals.

Besides, for the illumination by blue and green light in Figs. 6(a) and 6(b), respectively, we observe that the locations of maximum $m$ appear to be off-centered. In other words, although the parameters of the metasurface were selected as to maximize $P_{-1}^{r}$ for $\theta=60^{\circ}, \lambda_{0}=470 \mathrm{~nm}$, and $\lambda_{0}=532 \mathrm{~nm}$, respectively, the maximization of $m$ is obtained around $4^{\circ}$ and a few nanometers away from these values. On the other hand, the maximization of $m$ for yellow, orange, and red is achieved exactly at the specific values of $\lambda_{0}$ and $\theta$, which were originally used to optimize the anomalous reflection performance of the metasurface [cf. Figs. 6(c)-6(e) and Table 1].

\section{CONCLUDING REMARKS}

Anomalous reflection phenomena by a gradient dielectric metasurface composed of two periodically alternating lossless materials were investigated in the visible frequency range. The diffraction problem was solved by a highly efficient entire-domain integral equation methodology. The results of this methodology are compatible with energy conservation and physical intuition and have already been checked against the ones obtained with different techniques. Systematic optimizations were performed leading to the determination of the metasurface's width and period such that the power of the -1-reflected order is significantly enhanced, while the corresponding powers of the zero-reflected and -transmitted orders are annihilated. For certain frequency intervals, the powers of the -1 -reflected order can even reach $100 \%$. The materials of the metasurface's unit cell offering these remarkable characteristics can be ordinary dielectrics. The proposed optimized alldielectric structures can be used in several components and devices that use the mechanism of anomalous reflection to boost their efficiency. Their application range spans from wavefront manipulation, imaging, and beam steering to antireflection coatings, absorbers, and couplers.

The consideration of inclusions of different shapes per unit cell as well as of different duty cycles may assist further in achieving enhanced anomalous reflection effects for a wider 
range of frequencies and incident angles. Moreover, it is worth analyzing the generation of similar effects for the case of a transverse-magnetic (TM)-polarized plane incident wave. It is also interesting to examine choices of different realizable materials, composing the gradient metasurface, and determine respective optimal geometrical parameters in order to make the region of significant anomalous reflection more broadband.

\section{REFERENCES}

1. N. Yu, P. Genevet, M. A. Kats, F. Aieta, J.-P. Tetienne, F. Capasso, and Z. Gaburr, "Light propagation with phase discontinuities: generalized laws of reflection and refraction," Science 334, 333-337 (2011).

2. X. Ni, N. K. Emani, A. V. Kildishev, A. Boltasseva, and V. M. Shalaev, "Broadband light bending with plasmonic nanoantennas," Science 335, 427 (2011).

3. C. Argyropoulos, G. D'Aguanno, N. Mattiucci, N. Akozbek, M. J. Bloemer, and A. Alù, "Matching and funneling light at the plasmonic Brewster angle," Phys. Rev. B 85, 024304 (2012).

4. F. Monticone, N. M. Estakhri, and A. Alù, "Full control of nanoscale optical transmission with a composite metascreen," Phys. Rev. Lett. 110, 203903 (2013).

5. N. M. Estakhri, C. Argyropoulos, and A. Alù, "Graded metascreens to enable a new degree of nanoscale light management," Philos. Trans. R. Soc. A 373, 20140351 (2015).

6. A. Sihvola, "Enabling optical analog computing with metamaterials," Science 343, 144-145 (2014).

7. N. M. Estakhri and A. Alù, "Recent progress in gradient metasurfaces," J. Opt. Soc. Am. B 33, A21-A30 (2016).

8. A. Monti, A. Toscano, and F. Bilotti, "Exploiting the surface dispersion of nanoparticles to design optical-resistive sheets and Salisbury absorbers," Opt. Lett. 41, 3383-3386 (2016).

9. M. Fruhnert, A. Monti, I. Fernandez-Corbaton, A. Alù, A. Toscano, F. Bilotti, and C. Rockstuhl, "Tunable scattering cancellation cloak with plasmonic ellipsoids in the visible," Phys. Rev. B 93, 245127 (2016).

10. V. O. Byelobrov, T. L. Zinenko, K. Kobayashi, and A. I. Nosich, "Periodicity matters: grating or lattice resonances in the scattering by sparse arrays of subwavelength strips and wires," IEEE Antennas Propag. Mag. 57(6), 34-45 (2015).

11. Y.-J. Tsai, S. Larouche, T. Tyler, G. Lipworth, N. M. Jokerst, and D. R. Smith, "Design and fabrication of a metamaterial gradient index diffraction grating at infrared wavelengths," Opt. Express 19, 2441124423 (2011).

12. Y. Cui, Y. He, Y. Jin, F. Ding, L. Yang, Y. Ye, S. Zhong, Y. Lin, and S. $\mathrm{He}$, "Plasmonic and metamaterial structures as electromagnetic absorbers," Laser Photon. Rev. 8, 495-520 (2014).

13. A. A. Darweesh, S. J. Bauman, and J. B. Herzog, "Improved optical enhancement using double-width plasmonic gratings with nanogaps," Photon. Res. 4, 173-180 (2016).

14. S. Jahani and Z. Jacob, "All-dielectric metamaterials," Nat. Nanotechnol. 11, 23-36 (2016).

15. D. Lin, P. Fan, E. Hasman, and M. L. Brongersma, "Dielectric gradient metasurface optical elements," Science 345, 298-302 (2014).

16. Y. Kivshar and A. Miroshnichenko, "Meta-optics with Mie resonances," Opt. Photon. News 28(1), 24-31 (2017).

17. D. Ohana and U. Levy, "Mode conversion based on dielectric metamaterial in silicon," Opt. Express 22, 27617-27631 (2014).

18. L. Zhu, J. Kapraun, J. Ferrara, and C. J. Chang-Hasnain, "Flexible photonic metastructures for tunable coloration," Optica 2, 255-258 (2015).
19. S. Sun, K.-Y. Yang, C.-M. Wang, T.-K. Juan, W. T. Chen, C. Y. Liao, Q. He, S. Xiao, W.-T. Kung, G.-Y. Guo, L. Zhou, and D. P. Tsai, "High-efficiency broadband anomalous reflection by gradient metasurfaces," Nano Lett. 12, 6223-6229 (2012).

20. F. Aieta, P. Genevet, N. Yu, M. A. Kats, Z. Gaburro, and F. Capasso, "Out-of-plane reflection and refraction of light by anisotropic optical antenna metasurfaces with phase discontinuities," Nano Lett. 12 1702-1706 (2012).

21. S. Sun, Q. He, S. Xiao, Q. Xu, X. Li, and L. Zhou, "Gradient-index meta-surfaces as a bridge linking propagating waves and surface waves," Nat. Mater. 11, 426-431 (2012).

22. Y. Li, B. Liang, Z.-M. Gu, X.-Y. Zou, and J.-C. Cheng, "Reflected wavefront manipulation based on ultrathin planar acoustic metasurfaces," Sci. Rep. 3, 2646 (2013).

23. M. Khorasaninejad, F. Aieta, P. Kanhaiya, M. A. Kats, P. Genevet, D. Rousso, and F. Capasso, "Achromatic metasurface lens at telecommunication wavelengths," Nano Lett. 15, 5358-5362 (2015).

24. N. L. Tsitsas, N. K. Uzunoglu, and D. I. Kaklamani, "Diffraction of plane waves incident on a grated dielectric slab: an entire domain integral equation analysis," Radio Sci. 42, RS6S22 (2007).

25. N. L. Tsitsas, "Efficient integral equation modeling of scattering by a gradient dielectric metasurface," Eur. Phys. J. Appl. Metamater. 4, 3 (2017).

26. N. L. Tsitsas, D. I. Kaklamani, and N. K. Uzunoglu, "Integral equation analysis of coupling in symmetric grating assisted optical waveguides," J. Opt. Soc. Am. A 23, 1488-1502 (2006).

27. N. L. Tsitsas, D. I. Kaklamani, and N. K. Uzunoglu, "Rigorous integral equation analysis of nonsymmetric coupled grating slab waveguides," J. Opt. Soc. Am. A 23, 2888-2905 (2006).

28. M. Weber and D. L. Mills, "Interaction of electromagnetic waves with periodic gratings: enhanced fields and the reflectivity," Phys. Rev. B 27, 2698-2709 (1983).

29. P. M. Morse and H. Feshbach, Methods of Theoretical Physics, Part I (McGraw-Hill, 1953).

30. H. L. Bertoni, L.-H. S. Cheo, and T. Tamir, "Frequency-selective reflection and transmission by a periodic dielectric layer," IEEE Trans. Antennas Propag. 37, 78-83 (1989).

31. M. G. Moharam, E. B. Grann, D. A. Pommet, and T. K. Gaylord, "Formulation for stable and efficient implementation of the rigorous coupled-wave analysis of binary gratings," J. Opt. Soc. Am. A 12, 1068-1076 (1995).

32. https://en.wikipedia.org/wiki/Visible_spectrum (accessed January 25, 2017).

33. M. K. Yang, R. H. French, and E. W. Tokarsky, "Optical properties of Teflon® AF amorphous fluoropolymers," J. Micro/Nanolith. MEMS MOEMS 7, 033010 (2008).

34. D. E. Aspnes and A. A. Studna, "Dielectric functions and optical parameters of $\mathrm{Si}, \mathrm{Ge}, \mathrm{GaP}, \mathrm{GaAs}, \mathrm{GaSb}, \mathrm{InP}, \mathrm{InAs}$, and InSb from 1.5 to $6.0 \mathrm{eV}$," Phys. Rev. B 27, 985-1009 (1983).

35. http://refractiveindex.info/ (accessed January 11, 2017).

36. D. C. O'Shea, T. J. Suleski, A. D. Kathman, and D. W. Prather, Diffractive Optics Design, Fabrication and Test (SPIE, 2004).

37. K.-P. Chen, S.-C. Ye, C.-Y. Yang, Z.-H. Yang, W. Lee, and M.-G. Sun, "Electrically tunable transmission of gold binary-grating metasurfaces integrated with liquid crystals," Opt. Express 24, 16815-16821 (2016).

38. N. L. Tsitsas and N. K. Uzunoglu, "Scattering by a grating slab waveguide with regular plane regions grooves: integral equation modeling," IEEE Trans. Magn. 45, 1080-1083 (2009).

39. R. Petit, Electromagnetic Theory of Gratings (Springer, 1980).

40. J. Nakayama and A. Kashihara, "Energy balance formulas in grating theory," IEICE Trans. Electron. E86-C, 1106-1108 (2003). 